\title{
Three-Dimensional Particle Tracking Velocimetry using a Single Time-of-Flight Camera
}

\author{
S. E. Morris ${ }^{1 *}$, A. D. Goldman ${ }^{1}$, B. S. Thurow ${ }^{1}$ \\ ${ }^{1}$ Auburn University, Samuel Ginn College of Engineering, Auburn, USA \\ *sem0116@auburn.edu
}

\begin{abstract}
Time of Flight (ToF) cameras are a type of range-imaging camera that provides three-dimensional scene information from a single camera. This paper assesses the ability of ToF technology to be used for threedimensional particle tracking velocimetry (3D-PTV). Using a commercially available ToF camera various aspects of 3D-PTV are considered, including: minimum resolvable particle size, environmental factors (reflections and refractive index changes) and time resolution. Although it is found that an off-the-shelf ToF camera is not a viable alternative to traditional 3D-PTV measurement systems, basic 3D-PTV measurements are shown with large $(6 \mathrm{~mm})$ particles in both air and water to demonstrate future potential use as this technology develops. A summary of necessary technological advances is also discussed.
\end{abstract}

\section{Introduction}

In this work, the ability of a single time-of-flight ( $\mathrm{ToF}$ ) camera to perform three-dimensional particle tracking velocimetry (3D-PTV) measurements is explored. Many flow fields are 3D and unsteady in nature, and require advanced diagnostic techniques for quantitative measurements. Particle image velocimetry (PIV) and particle tracking velocimetry (PTV) are two of the most common flow diagnostic tools (Aguirre-Pablo et al., 2017). PIV returns an Eularian perspective of the flow field, wherein velocity information does not rely on individual particle identification. The most recent major development in PIV is that of tomographic PIV, wherein the instantaneous three-component velocity field is measured from different angles to create the multi-dimensional perspectives necessary for 3D measurement and volumetric reconstructions (Elsinga et al., 2006). In contrast, PTV yields Lagrangian perspective of the flow field as individual particles are tracked through space (Shnapp et al., 2019).

A major drawback of modern 3D-PIV/PTV techniques is that they can be cost-prohibitive for research laboratories. These techniques traditionally require significant laboratory space and the use of multiple research-level cameras, a synchronization unit, and a high-powered laser. Furthermore, trained personnel and personal protective equipment are needed due to the risks of laser use. As a result, recent advances in 3D flow measurements have focused on moving towards single-camera systems for three-dimensional, three-component velocity field measurements. Such single-camera systems for these measurements include plenoptic (light field) cameras, color-coded illumination, scanning laser sheets/ volumes and image splitters (for further details and examples see Aguirre-Pablo et al. (2019)). The use of range-imaging cameras such as LIDAR and ToF technology as a flow diagnostic tool has been increasing, for example LIDAR-based PIV has been shown to successfully analyze very large slow-moving solid objects like glaciers (Telling et al., 2017), and ToF cameras have been used in combination with traditional PIV for data enhancement of seeded flows (Augère et al., 2017). ToF cameras have also been used to evaluate planar seeded flow, with independent camera and light sources (Paciaroni et al., 2018). Recently, single camera 3D-PTV has been achieved by Romano et al. (2021) using a two-view splitter and Rossi and Marin (2020) by taking advantage of astigmatic aberrations.

This work explores the use of a single off-the-shelf correlation ToF camera as a flow diagnostic tool for 3D-PTV measurements. In addition to being a single-camera singular port system, this technique eliminates the need for separate volume illumination via a laser or other means. This paper will assess the ability of a ToF camera to conduct simple 3D-PTV measurements, and evaluate the current state of technology of these cameras for this application. An overview of ToF technology is discussed in $\$ 2$, and Experimental Methods 
are presented in \$3. The primary aspects of 3D-PTV that are assessed in this paper include: the flow field environment, the fluid medium, minimum resolvable particle size, particle type and $(x, y, z, t)$ resolution; these are presented in $\$ 4$. A further discussion of the state of ToF technology is given in $\$ 5$, and conclusions follow in $\$ 6$.

\section{Time of Flight Camera Technology}

ToF cameras are a type of range-imaging camera that work by measuring the time it takes for light emitted by the camera to reflect off an object and return to the camera's sensor (Hansard et al., 2012). In doing so, the depth of objects in a scene are resolved. Today, these cameras are commonly used in digital mapping (Dowman, 2004), altimetry (Hu et al., 2007), oceanography (Churnside, 2013), construction (Wang et al. 2015) and autonomous vehicles (Wang et al., 2017).

The ToF camera light source is typically an infra-red LED or laser diode, and can be either pulsed or continuous. In pulsed modulation, the time taken for the light to travel is directly measured. In continuous wave modulation (also known as Indirect or Correlation ToF cameras), the phase-shift between the emitted light and reflected light is measured. These signals commonly take the form of sinusoidal or square wave signals. This phase-shift is proportional to the distance travelled, $D$ :

$$
D=\frac{1}{2} \frac{c \Delta \phi}{2 \pi f}
$$

where $c$ is the speed of light, $f$ is the modulation frequency and $\Delta \phi$ is the phase difference.

In correlation ToF cameras, the depth (distance) of objects is determined by an image sensor (typically CMOS) that measures these phase-shifts (He and Chen, 2019; Kadambi and Raskar, 2017). As the modulation frequency is increased, the depth resolution and accuracy is also increased (Kadambi and Raskar, 2017). In contrast to a scanning mechanism (such as scanning LIDAR), each pixel in a ToF image simultaneously measures the distance between the camera and the corresponding object seen by a given pixel (Hansard et al. 2012). This information is stored as a depth map, wherein each $(\mathrm{x}, \mathrm{y})$ location is prescribed a depth (z). This is commonly output as 3D point cloud data.

In comparison to traditional flow diagnostic cameras, ToF cameras provide several advantages. As they are self-contained, they provide 3D field information via a singular port, lightweight unit. These cameras have in recent years become relatively inexpensive, and eliminate the need for volume illumination as their light source is in-built.

In this paper, an off-the-shelf ToF 3D Camera is used to perform exploratory 3D-PTV experiments. The camera has a resolution of $0.3 \mathrm{MP}(640 \times 480 \mathrm{px})$, and a field of view of $59^{\circ} \times 45^{\circ}$ with a focal length of $6 \mathrm{~mm}$. The lens is in-built, and would require custom modifications to change. The camera has two working modes, near mode ( $1.5 \mathrm{~m}$ depth working distance) and far mode (6m depth working distance). These have final working areas of $1.697 \mathrm{~m} \times 1.243 \mathrm{~m}$ and $6.789 \mathrm{~m} \times 4.971 \mathrm{~m}$ respectively. In this work, only the near mode will be considered. The precision of the camera decreases at further $z$ depths: at $z=0.5 \mathrm{~m}$ the precision is $0.69 \mathrm{~mm}$ and at $z=1.5 \mathrm{~m}$ the precision is $3.11 \mathrm{~mm}$ (LucidVision, 2019). In this mode, the camera operates at a frame rate of 30fps. The exposure time can be chosen as $1000 \mu \mathrm{s}$ or $250 \mu \mathrm{s}$. The camera uses a Sony IMX556 CMOS Sensor and four Vertical Cavity Surface Emitting Laser (VCSEL) diodes at $850 \mathrm{~nm}$ with a modulation frequency of $100 \mathrm{MHz}$ and power of $2.2 \mathrm{~mW}$ (continuous wave modulation). This sensor measures phase shifts by use of a current assisted photonic demodulator (CAPD) pixel structure. Within each pixel's photodiode, light is converted into electrons and the CAPD uses an alternating voltage to pull electrons to alternating detector junctions. The primary junction samples light at the same frequency it is transmitted and the secondary junction samples light $180^{\circ}$ out of phase to calculate the phase shift (LucidVision, 2020).

\section{Experimental Methods}

To evaluate the ability of an off-the-shelf ToF camera to track particles for PTV measurements, a commercially available ToF camera in near-mode $(1.5 \mathrm{~m})$ with $250 \mu$ s exposure is used. Experiments were chosen to evaluate different aspects that contribute to the effectiveness and accuracy of 3D-PTV, such as scene reflections, different fluid mediums, the minimum resolvable particle size, particle types and $(x, y, z, t)$ resolution.

To measure the minimum resolvable particle size, an empty 5-sided glass tank is used as shown in figure 1 (a). In open-air experiments, the ToF camera is pointed towards the empty side of the tank to eliminate any 
(a)

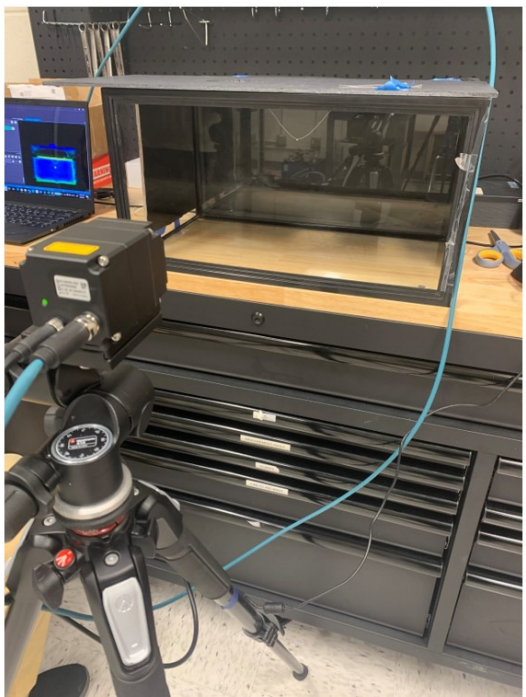

(b)

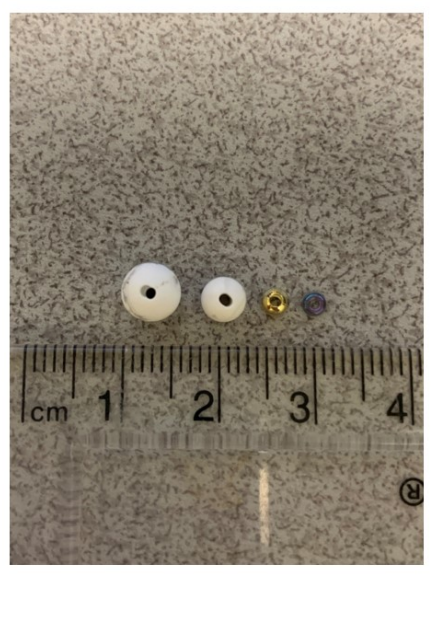

(c)

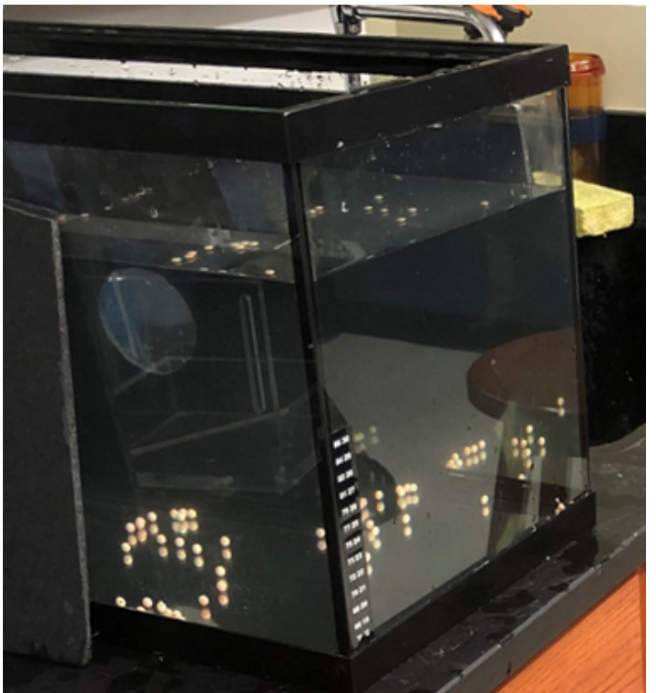

Figure 1: (a) 5-sided glass tank used for open-air experiments, with a fishing line holding a single stationary bead. This set-up was further developed to include matte black felt lining on the inside of the tank (not shown). (b) Example of plastic "seed beads" used in experiments. Silver seed beads (not shown) also ranged in size from $1 \mathrm{~mm}-6 \mathrm{~mm}$. (c) Water tank setup with "trout bait" tracer particles.

errors arising from refractive index changes. For the configuration shown in figure 1 ( $(a)$, it was ascertained that having black card on the outside of the tank as shown was not sufficient to remove reflections of the VCSEL diodes from the inner glass wall. As such, matte black felt material was placed inside the tank walls (not shown). This provided a number of benefits: the black felt removed any reflections from the walls, it provided a constant depth (z) background within the field of view, and it removed any aliasing arising from the camera measuring objects outside the region of interest (see \$4.1).

Initially, different sized "seed beads" were held stationary, suspended via fishing line (see figure 1) in the camera's field of view to determine the minimum resolvable particle size. Seed beads are commonly used in jewelry making and can come in sizes as small as $O(1 \mathrm{~mm})$. They are usually toroidal or cylindrical in shape, and have a hole through their center. Two types of seed beads were used: plastic seed beads ranging in diameter from $2 \mathrm{~mm}-6 \mathrm{~mm}$ as shown in figure $1(b)$, and silver beads of diameter $1 \mathrm{~mm}-6 \mathrm{~mm}$ (not shown).

Although it was determined that the camera could detect stationary particles as small as $2 \mathrm{~mm}$, for PTV experiments the seed bead size was kept as $6 \mathrm{~mm}$ unless otherwise stated. To determine the ability of the camera to track multiple moving particles in $(x, y, z, t)$, three seed beads were initially dropped in the middle of the camera's field of view and tracked as they dropped and bounced in front of the camera due to gravity. Although this showed the dynamic motion of the beads, due to the limited frame rate of the camera (30fps) it was determined necessary to slow the particles' velocities to properly assess the camera's ability to track the particles. In order to achieve this, a set of fishing lines both parallel and perpendicular to the camera were set such that a $6 \mathrm{~mm}$ particle could slide along the line in predictable motions. The angle that the fishing line made with the horizontal (ground) controlled the velocity of the beads. PTV measurements were calculated in MATLAB, wherein a particle's location in the point cloud was found via a peak search function in the measurement field of view. The velocity of the particle is calculated via a (two-point) forward time difference.

Following air experiments, the tank was filled with water with $6 \mathrm{~mm}$ "trout bait eggs" used as tracer particles to determine the ability of the camera to track moving particles in water (see figure $1(c)$. The trout bait have a slower settling velocity than seed beads, and so were able to stay briefly suspended in manually perturbed water. It must be noted that in practice this experiment would require a depth calibration, as refractive index changes occur when viewing through the glass wall and water, however as a proof-ofconcept experiment these calibrations were not performed. The back wall of the tank was lined with matte black felt to minimize reflections.

In addition to solid particles, a set of experiments were performed to determine whether the camera could distinguish soap bubbles in the same manner as solid particles. A LaVision helium-filled soap-bubble (HFSB) generator was used to generate $300 \mu \mathrm{m}$ neutrally buoyant particles, and a standard children's bubble wand was used to generate bubbles on the order of $1 \mathrm{~cm}-5 \mathrm{~cm}$. 


\section{Results and Discussion}

\subsection{Environment}

As mentioned in $\$ 3$, an idealized environment was necessary for reliable PTV experiments. As such, the background of the flow field of view needed to be controlled. The reasons for this are two-fold: to prevent aliasing, and to prevent reflections. Aliasing occurs in ToF cameras if the time taken for the emitted light to return to the sensor is longer than the period of the modulated light. This occurs when the distance to an object differs in phase by 360 degrees to the phase shift, and becomes indistinguishable (Gokturk et al., 2004). The unambiguous distance, $D_{\text {unamb. }}$, (Melexis, 2020) can be calculated by:

$$
D_{\text {unamb. }}=\frac{c}{2 f}
$$

For the camera operating in near-mode $(f=100 \mathrm{MHz})$, this is equal to $1.5 \mathrm{~m}$. By providing a constantdepth background within the unambiguous range, the camera will not report any aliasing of the background that may interfere with particle recognition or detection. In future ToF PTV work, it is recommended that experimentalists provide a non-reflective, constant depth background for optimal measurements.

With respect to preventing reflections, it is established that the colour, type of material and distance from camera can all influence the depth error recorded by a ToF camera (He et al., 2017). Shiny surfaces or objects with very high reflectivity can cause the pixel(s) to saturate, losing phase information and invalidating the depth calculation (LucidVision, 2019). As such, if the laser power of a ToF camera is increased to detect smaller particles (Raffel et al., 2018), care must be taken to ensure that the background or particles themselves do not over-saturate. Similarly, dark or matte surfaces are good absorbers and hence poor reflectors of light: a black surface will absorb most of the photons, leading to a low depth accuracy of black surfaces (Baek et al., 2020).

As aforementioned, it was ascertained early that a glass-walled tank would reflect the four VCSEL diodes back in the 3D depth map (and in fact over-saturate such that a depth could not be calculated). This is shown in figure $2(a)$, wherein four black circles corresponding to the four VCSEL diode reflections can be seen (indicated by the white circle). Black matte felt material was added to the inner walls of the tank to remove these reflections; this is shown in figure $2(b)$.

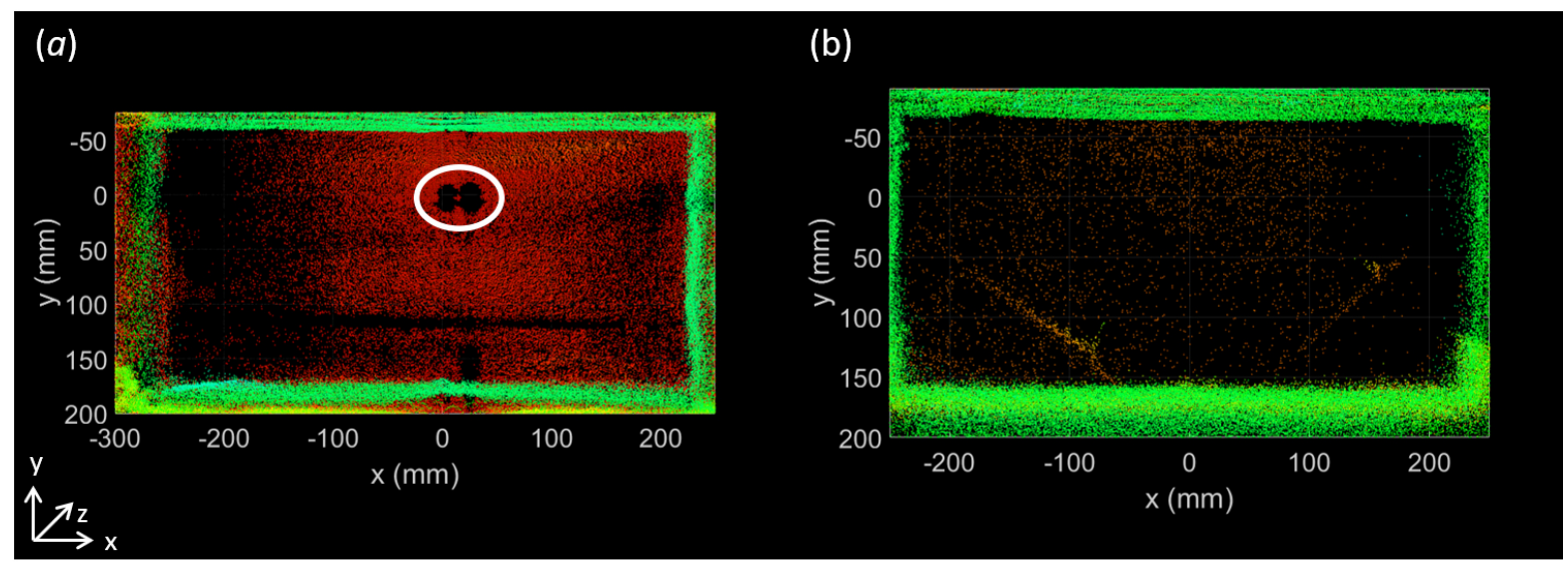

Figure 2: (a) Open-air tank, showing the reflection of the four VCSEL diodes (shown in white circle). The reflections are over-saturated and do not provide depth information. (b) Open-air tank with matte black felt backing. No reflections are observed, and the backing provides a constant-depth background. Colour indicates $z$ depth, where green is closer to the camera and red is further away.

\subsection{Fluid Medium and Distortions}

ToF technology works on the ability of the camera to emit a modulated light signal and receive it at its sensor. As light passes through different mediums, it will refract (Snell's law) due to the change in light speed. The implication of this in ToF technology is decreased or changed location accuracy. This was 
observed experimentally by holding a piece of acrylic in front of the camera, and measuring the change in a stationary object's distance. It is therefore recommended that depth calibrations be performed for ToF 3D-PTV experiments that take place through glass or acrylic walls, or pass through water.

\subsection{Particle Seeding}

It is well established that the particle seeding of a flow field is of importance when performing PIV/PTV measurements. It is typically desirable that the particles be $O(\mu \mathrm{m})$ or smaller and neutrally buoyant with the fluid. As such, it was necessary to ascertain the camera's minimum resolvable particle size in air. Initially, solid seed bead particles were held stationary in the matte black tank in front of the camera. For a set of silver seed beads, the camera's minimum resolvable stationary particle size was found to be $2 \mathrm{~mm}$; when the particle was allowed to drop with gravity in front of the camera, the minimum resolvable size increased to $3 \mathrm{~mm}$. In near mode, this minimum resolvable particle size is comparable to the pixel size. As such, for particles smaller than a pixel, the signal returned to the pixel will be a combination of both the particle and any residual background light. It must also be noted here that the colour of the particle plays an important role in its detectability. As the ToF camera operates using an infra-red camera, it has difficulty establishing the location of black or red particles (see \$4.1). It is suggested that white or reflective (e.g. silver) particles are used for maximum detectability. An example of the depth map (3D point cloud) for a single solid particle is shown in figure $3(a)$. A test of particle size was not performed in water, however figure $3(b)$ shows the depth map for a collection of $6 \mathrm{~mm}$ plastic particles (approximately 25 particles/gallon) in water.

In addition to solid particles, the ability of the camera to detect soap bubbles was tested due to the increased popularity of using HFSB particles as fluid tracers. $300 \mu m$ neutrally buoyant helium-filled soapbubbles generated by a LaVision HFSB Generator were recorded with the ToF camera and were found to be
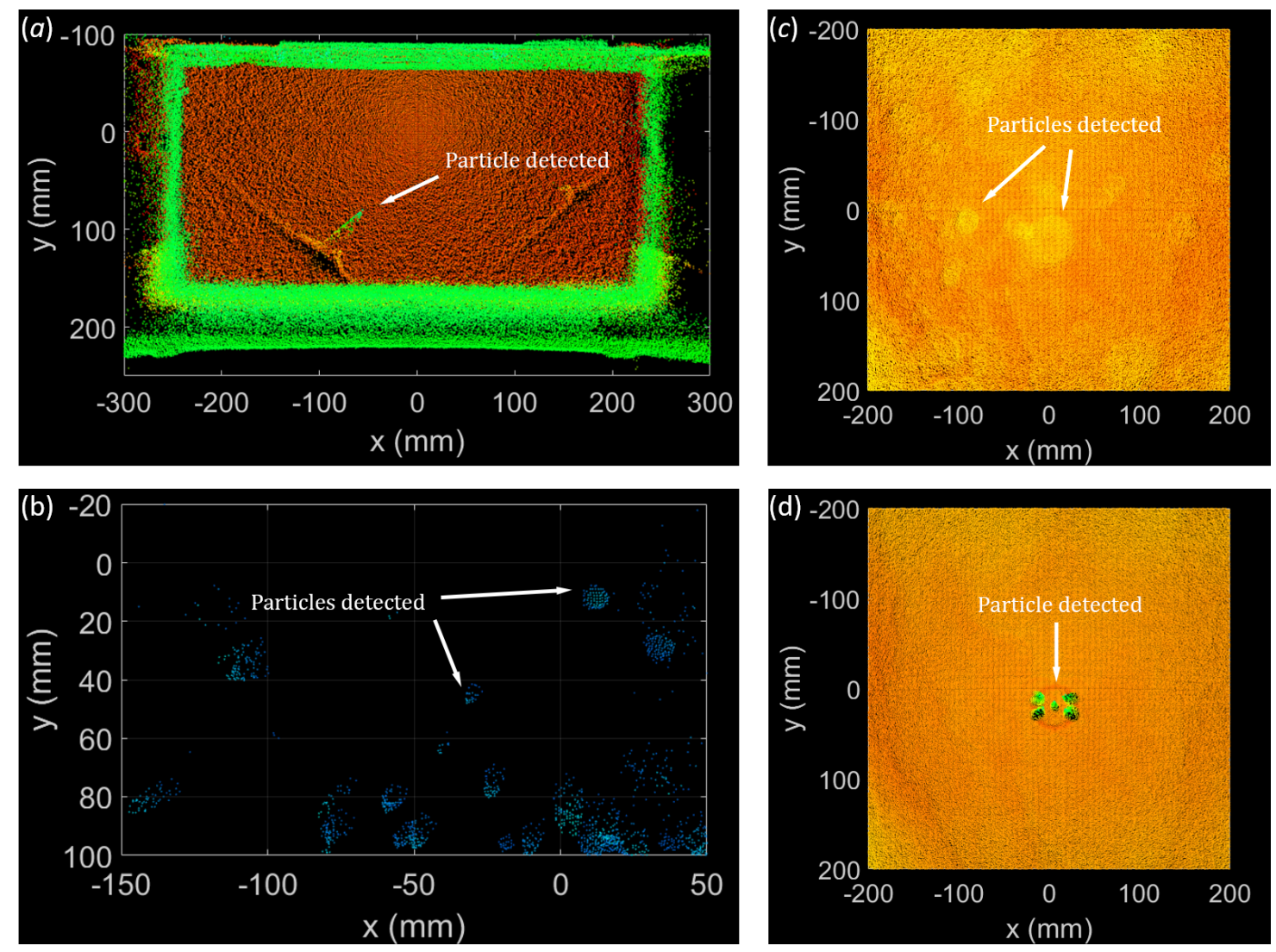

Figure 3: (a) Single plastic particle detected in open-air experiment. (b) Multiple "trout bait" particles detected in water. (c) HFSB particles detected in open-air, with no depth resolution. (d) Soap bubble detected in open-air. VCSEL laser diode reflections interfere with depth resolution. Colour indicates $z$ depth, where green is closer to the camera and red is further away. 
incapable of being resolved, even when the density of HFSB particles was decreased. Figure $3(c)$ shows the depth map of a set of HFSB particles. Large circles indicate a soap bubble very close to the camera, and small circles further away. However, as can be seen, the camera is unable to determine any depth changes between them (all are the same depth colour). Following this, large bubbles $(1 \mathrm{~mm}-5 \mathrm{~mm})$ were blown in front of the camera via a children's bubble wand. In this case, a new problem arose: as can be seen in figure $3(d)$, four distinct points can be seen on the bubble. These four distinct points represent a reflection from each of the four VCSEL diodes. This reflection made the shape and distance of the bubble difficult to resolve; this could potentially be an issue in the future using HFSB bubbles, however may be mitigated due to their small size.

Currently, it is evident that the ToF camera cannot resolve particle sizes required for traditional PTV measurements. As discussed in $\$ 2$, the depth resolution and camera accuracy is increased as the modulation frequency of the light source is increased. In order to detect traditional PTV/PIV particles, a ToF camera will need a much higher modulation frequency (see $\$ 5$ for further details on the 'GHz Gap' (Kadambi and Raskar, 2017)).

It is also noted that ToF cameras act as a single line of sight camera. Using a single line of sight camera introduces difficulty in 3D-PTV measurements, as any object behind another will not be present in the depth map. This is because each $(x, y)$ location can only determine a single $(z)$ depth. As such, there is a loss of information each time a particle passes behind another, limiting particle tracking in cases with crossover interactions. In future work, it is suggested that predictive algorithms in crossover interactions and a careful choice in particle seeding density be used.
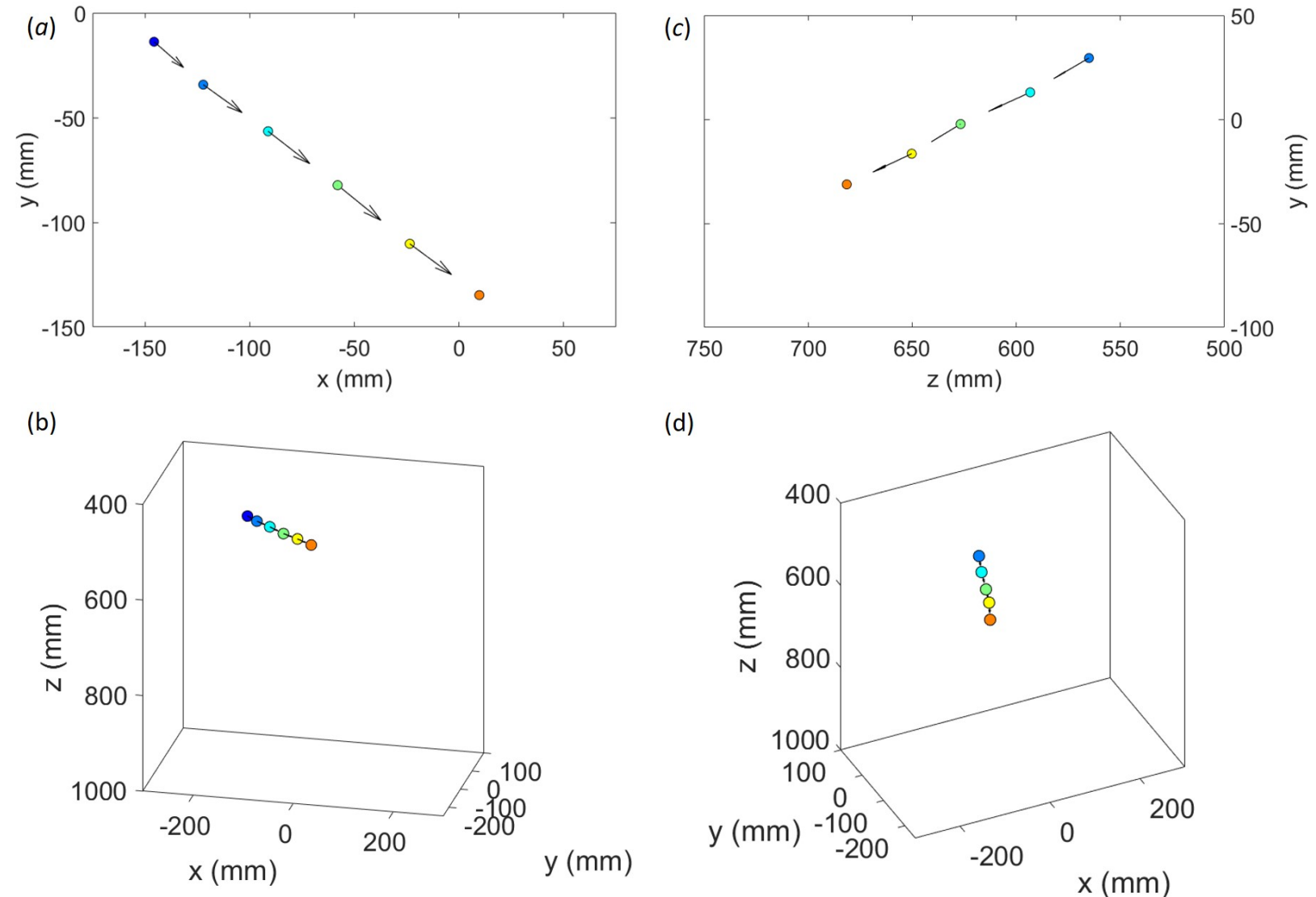

(d)

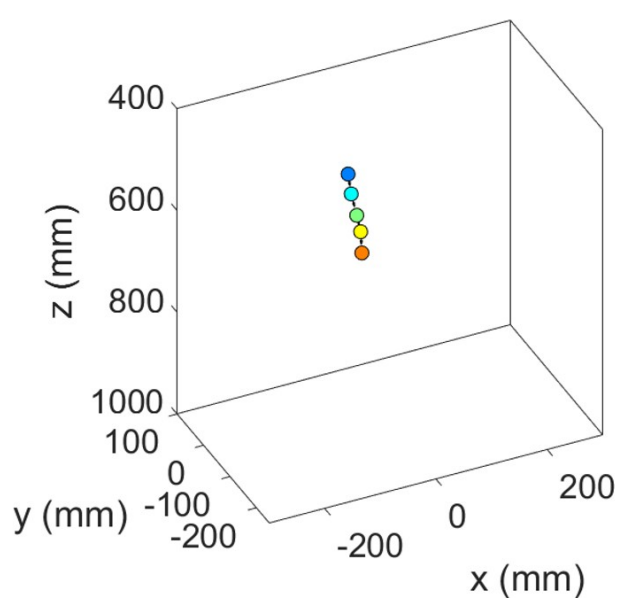

Figure 4: (a) Single particle travelling in open-air parallel to the camera face, shown in the $(x, y)$ plane, and (b) in 3D. (c) Single particle travelling in open-air perpendicular to the camera face, shown in the $(y, z)$ plane and $(d)$ in 3D. Time steps are indicated from cool (blue) to warm (orange). 


\subsection{Volumetric and Time Resolution}

In this section, the results of basic $2 \mathrm{D}$ and $3 \mathrm{D}$ motion experiments using the ToF camera are reported. It must be noted that these are basic representations of PTV experiments using $6 \mathrm{~mm}$ particles at 30fps, limited by the camera's ability: for real flow experiments, improvements must be made in ToF camera technology to allow for particle tracking of typical flow seeding particles (1-2 orders of magnitude smaller than used in the current experiments). Furthermore, frame rate and exposure controls would be required to expand the camera's capability to track both high and low Reynolds number flows.

\subsubsection{Planar Motion}

To evaluate the ToF camera's ability to track simple 2D motion, a series of $2 \mathrm{D}$ motion experiments were conducted in the matte black tank in air (camera viewing through the open face). The first test was the ability of the camera to track both in-plane $(x, y)$ and out-of-plane $(z)$ motions. To achieve this, a fishing line was set up firstly in the $(x-y)$ plane (motion parallel to the camera face or in-plane), and a $6 \mathrm{~mm}$ bead allowed to slide along it. As aforementioned, this 'sliding' technique was used to slow down the solid particle motion, as we were restricted to 30fps image acquisition. Figure $4(a)$ and $(b)$ shows the superposition of multiple point cloud depth images, with the particles detected shown (increasing time is indicated as particles change colour from cool to warm). The camera is able to accurately track the motion of the bead in $(x-y)$, with minimal $(z)$ displacements. Similarly, a fishing line was set up in the $(y-z)$ plane (motion parallel to the camera face or out-of-plane). This is shown in figure $4(c)$ and $(d)$. The ToF camera well captures the out-of plane $(z)$ motion, with minimal $(x)$ displacements.

\subsubsection{D Motion}

Following the success of the camera at tracking a single particle in air in a controlled environment, the camera was tested at tracking multiple particles in 3D. A simple experiment was initially conducted in air, dropping three beads and allowing them to bounce in front of the camera. The raw depth image (multiple time steps superimposed on each other) with particle identification is shown in figure 5 (a). Time steps are indicated from cool (blue) to warm (orange). The 3D-PTV field is shown in $(b)$. As can be seen, the lack of frame rate control becomes an issue in this case. Due to the low time resolution, we are unable to resolve the bouncing motion sufficiently. As such, errant velocity vectors arise when a particle is known to be travelling downward, but the following time step particle has bounced above it. Nonetheless, the camera is able to track the particle locations reasonably well.
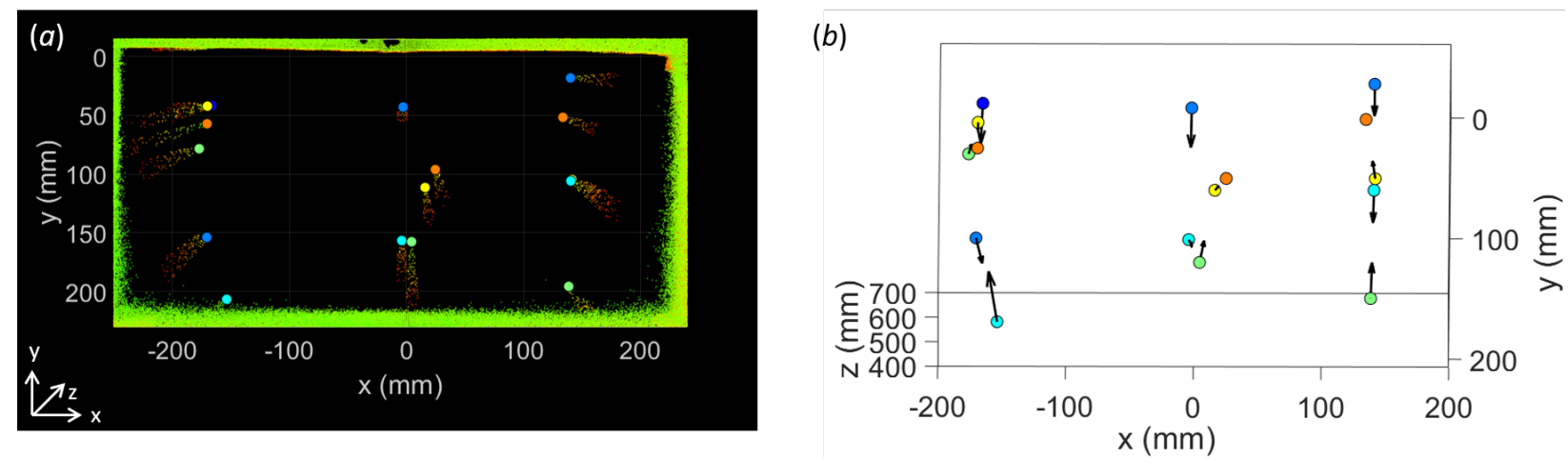

Figure 5: (a) Point cloud image of multiple time frames showing three beads dropping due to gravity in open air. (b) 3D-PTV field of the three particles. Time goes from cool (blue) to warm (orange).

As the frame rate of the camera limited the open-air experiments, 3D-PTV was also conducted in a water tank using $6 \mathrm{~mm}$ "trout bait" particles. The water was randomly perturbed, and the motions tracked as the particles moved in 3D before settling. It must be noted that these experiments were not calibrated to account for refractive index changes, but were rather performed as a proof-of-concept. Figure 6 ( $a$ ) shows the superposition of multiple point cloud depth images, with the particles detected shown (increasing time is indicated as particles change colour from cool to warm). The 3D-PTV field is shown in figure $6(b)$. The 3D-PTV field demonstrates the potential of a single ToF camera to accurately track particle motion in 3D, 
however the limitations of particle size and camera frame rate must be improved before a 'real' flow can be accurately analysed with this technique.
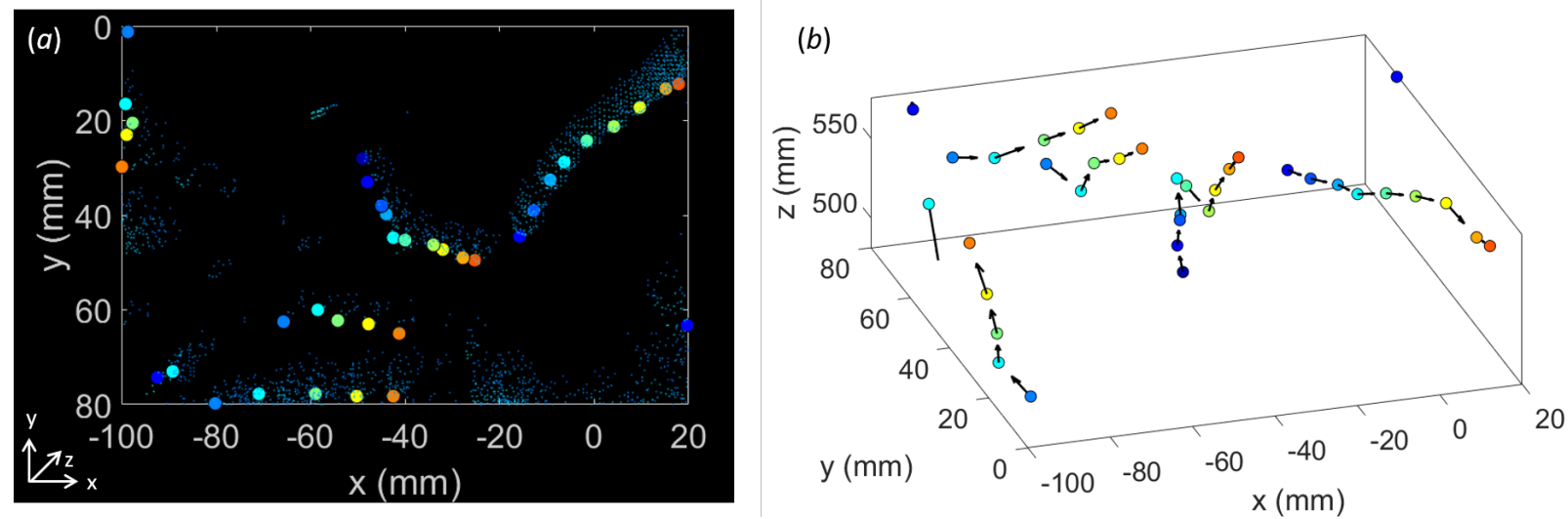

Figure 6: (a) Point cloud image of multiple particles being manually perturbed in water. (b) 3D-PTV field of the manually perturbed particles. Time goes from cool (blue) to warm (red).

\section{Assessment of the State of Technology}

The results of this study have shown that it is possible to perform multi-particle 3D-PTV using ToF technology. At current standing however, the 3D-PTV fields obtained using a commercially available ToF camera are severely limited. The following advancements are necessary for ToF PTV to be a viable alternative to traditional PTV:

1. A primary limitation of correlation ToF cameras for 3D-PTV is the depth resolution achievable with off-the-shelf cameras (the maximum precision of the current configuration is $0.69 \mathrm{~mm}$ at $z=0.5 \mathrm{~m}$ ), and hence the minimum particle size capable of being detected. To improve depth resolution, higher modulation frequencies are required. However, the current state of image sensors are unable to detect these frequencies: this is referred to as the "GHz gap" in ToF Technology (Kadambi \& Raskar 2017). One method suggested to improve the depth resolution of ToF cameras - without requiring $\mathrm{GHz}$ modulation frequencies - is to incorporate heterodyning using a cascaded modulation scheme; a prototype demonstrates $3 \mu \mathrm{m}$ precision over a range of $2 \mathrm{~m}$ (Kadambi \& Raskar 2017).

2. The minimum detectable particle size is also related to the power output of the light source, as the particle image intensity (reflected light) is directly proportional to the scattered light power (Raffel et al., 2018). Increasing the light power may however also increase background reflections, and there may be a critical power limit to illuminate small particles with minimal background reflections. Recently, Qiu et al. (2019) have reported achieving high-efficiency $905 \mathrm{~nm}$ pulsed lasers with a power as high as 150W for long-range LIDAR applications; this is orders of magnitude higher than the VCSEL diodes $(2.2 \mathrm{~mW})$ built-in to the ToF camera used in these experiments.

3. As ToF technology works on light line of sight, errors are introduced when there are refractive index changes or reflective/absorbing surfaces. In the case of refractive index changes, calibrations must be taken prior to experiments to account for these changes. In the case of reflective/absorbing surfaces, this limits the experimental configurations: light-coloured or reflective particles should be used for optimal detectability, and the flow field background should be matte black so as not to interfere with measurements and prevent aliasing.

4. As with other single camera flow diagnostic tools, a single line of sight camera will only provide a single perspective and so overlapping objects are not identifiable. This sets a limit on the particle seeding density, as the quality of PTV results will deteriorate with increased particle numbers (Cierpka et al., 2013). Pre-existing solutions that can be applied include adding additional cameras, or applying advanced time-resolved particle tracking algorithms. 
5. The ToF camera used in these experiments is limited by its fixed frame rate (30fps). For this method to be applicable to a wide range of Reynolds number flows, the time resolution must be increased and adjustable. Similarly, increased exposure control will be required at higher Reynolds numbers to prevent particle streaking.

\section{Conclusions}

ToF cameras have the potential to be used in the future as a single camera flow diagnostic tool for 3D-PTV measurements. However, given the current state of technology in common, off-the-shelf cameras, this is not yet a viable alternative for traditional 3D-PTV measurement systems.

Using a commercially available ToF camera, ToF technology was assessed in the context of 3D-PTV. The primary issues that are discussed that arose using this method include: particle size recognition, particle type recognition, refractive index changes, time resolution, and reflective/absorbing materials. Despite these challenges, using multiple $6 \mathrm{~mm}$ beads this paper shows basic 3D-PTV fields obtained from dropping particles (air) and from randomly disturbing the flow field (water) at 30fps.

\section{Acknowledgements}

The authors would like to acknowledge the members of the Advanced Flow Diagnostics Laboratory and the Applied Fluid Research Group in the Department of Aerospace Engineering at Auburn University.

\section{References}

Aguirre-Pablo AA, Alarfaj MK, Li EQ, Hernández-Sánchez JF, and Thoroddsen ST (2017) Tomographic particle image velocimetry using smartphones and colored shadows. Scientific Reports 7

Aguirre-Pablo AA, Aljedaani AB, Xiong J, Idoughi R, Heidrich W, and Thoroddsen ST (2019) Singlecamera 3d ptv using particle intensities and structured light. Experiments in Fluids 60

Augère B, Goular D, Valla M, Goular Y, Dolfi-Bouteyre A, Monnier J, Gilliot A, and Gallas Q (2017) Mise en œuvre d'un lidar doppler $1.5 \mu \mathrm{m}$ et de la spiv pour la caractérisation d'écoulements sur une plateforme d'appontage d'une maquette simplifiée de frégate en soufflerie. 23ème Congrès Français de Mécanique 23

Baek ET, Yang HJ, Kim SH, Lee G, and Jeong H (2020) Distance error correction in time-of-flight cameras using asynchronous integration time. Sensors (Basel, Switzerland) 20

Churnside JH (2013) Review of profiling oceanographic lidar. Optical Engineering 53

Cierpka C, Lütke B, and Kähler CJ (2013) Higher order multi-frame particle tracking velocimetry. Experiments in Fluids 54:1-12

Dowman I (2004) Integration of lidar and ifsar for mapping. the. in International Archives of the Photogrammetry, Remote Sensing and Spatial Information Sciences, Vol. XXXV, Part B2

Elsinga GE, Scarano F, Wieneke B, and Van Oudheusden BW (2006) Tomographic particle image velocimetry. Experiments in Fluids 41:933-947

Gokturk S, Yalcin H, and Bamji C (2004) A time-of-flight depth sensor - system description, issues and solutions. in 2012 IEEE Computer Society Conference on Computer Vision and Pattern Recognition Workshops. volume 4. page 35. IEEE Computer Society, Los Alamitos, CA, USA

Hansard M, Lee S, Choi O, and Horaud R (2012) Time of Flight Cameras: Principles, Methods, and Applications. SpringerBriefs in Computer Science. Springer

He Y and Chen S (2019) Recent advances in 3d data acquisition and processing by time-of-flight camera. IEEE Access 7:12495-12510 
He Y, Liang B, Zou Y, He J, and Yang J (2017) Depth errors analysis and correction for time-of-flight (tof) cameras. Sensors 17:92

Hu Y, Vaughan M, Tepte C, Weimer C, Beherenfeld M, Young S, Winker D, Hostetler C, Hunt W, Kuehn R, Flittner D, Cisewski M, Gibson G, Lin B, and Macdonnell D (2007) Elevation information in tail (eit) technique for lidar altimetry. Optics express 15:14504-15

Kadambi A and Raskar R (2017) Rethinking machine vision time of flight with ghz heterodyning. IEEE Access 5:26211-26223

LucidVision (2019) Helios General Technical Reference. Lucid Vision Labs

LucidVision (2020) Better Time of Flight: Sony DepthSense 3D Sensor Explained. Lucid Vision Labs

Melexis (2020) Time-of-flight (ToF) basics. Melexis Inspired Engineering

Paciaroni ME, Chen Y, Lynch KP, and Guildenbecher DR (2018) Backscatter particle image velocimetry via optical time-of-flight sectioning. Opt Lett 43:312-315

Qiu Y, Xie Y, Wang W, Liu W, Kuang L, Bai X, Hu M, and Ho J (2019) Ultra-high-power and high-efficiency 905nm pulsed laser for lidar. in 2019 IEEE 4th Optoelectronics Global Conference (OGC). pages 32-35

Raffel M, Kähler C, Willert C, Wereley S, Scarano F, and Kompenhans J (2018) Particle Image Velocimetry: A Practical Guide. Springer. 3rd edition

Romano M, Alberini F, Liu L, Simmons M, and Stitt E (2021) Lagrangian investigations of a stirred tank fluid flow using 3d-ptv. Chemical Engineering Research and Design 172:71-83

Rossi M and Marin A (2020) Single-Camera 3D PTV Methods for Evaporation-Driven Liquid Flows in Sessile Droplets. pages 225-236

Shnapp R, Shapira E, Peri D, Bohbot-Raviv Y, Fattal E, and Liberzon A (2019) Extended 3d-ptv for direct measurements of lagrangian statistics of canopy turbulence in a wind tunnel. Scientific Reports 9

Telling JW, Glennie C, Fountain AG, and Finnegan DC (2017) Analyzing glacier surface motion using lidar data. Remote Sensing 9

Wang H, Wang B, Liu B, Meng X, and Yang G (2017) Pedestrian recognition and tracking using 3d lidar for autonomous vehicle. Robotics and Autonomous Systems 88:71-78

Wang J, Sun W, Shou W, Wang X, Wu C, Chong HY, Liu Y, and Sun C (2015) Integrating bim and lidar for real-time construction quality control. Journal of Intelligent and Robotic Systems 79:417-432 